\title{
A Study on Multiple Barcode Detection from an Image in Business System
}

\author{
Atiqul Islam Chowdhury \\ Department of Computer Science \\ \& Engineering \\ Ahsanullah University of Science \\ and Technology \\ Dhaka, Bangladesh
}

\author{
Mushfika Sharmin Rahman \\ Department of Computer Science \\ \& Engineering \\ Ahsanullah University of Science \\ and Technology \\ Dhaka, Bangladesh
}

\author{
Nazmus Sakib \\ Department of Computer Science \\ \& Engineering \\ Ahsanullah University of Science \\ and Technology \\ Dhaka, Bangladesh
}

\begin{abstract}
The pillar of automatic identification is Barcode technology which is used comprehensively in real time applications with various types of codes. The different types of codes and applications sometimes faces special problems, so the improvement of the effectiveness must be done persistently. This paper's proposal mainly deals with multiple product's barcode to be detected simultaneously. The proposed algorithm which will have implementation for supershop billing system and inventory management. The method will recognize the barcodes using image processing. Images will be taken using mobile camera sensor. It will detect 1D barcodes such as EAN-13 barcodes, Code-128 barcodes, 2D barcodes such as QR codes. Moreover, it will be angle invariant, requires less user interaction than usual and can be executed on available computers. This model helps consumers to minimize the time for the billing system in shopping. We have implemented this in Python IDE using OpenCV library.
\end{abstract}

\section{Keywords}

Image processing, barcode, EAN-13, QR code, ZBar.

\section{INTRODUCTION}

As consumers, barcodes and barcode scanners are relatively familiar concept: purchasing from any retail store, renting a car, attending major events, flying, and even going to the doctor. They're in our social media apps and on store windows. A barcode is an optical, machine-readable, representation of data; the data usually describes something about the object that carries the barcode. It is a visual description of information in the form of bars and spaces on a surface [1]. Traditional barcodes systematically represent data by varying the widths and spacing of parallel lines, and may be referred to as linear or one-dimensional (1D). Later, twodimensional (2D) variants were developed, using rectangles, dots, hexagons and other geometric patterns, called matrix codes or 2D barcodes, although they do not use bars as such. Initially, barcodes were only scanned by special optical scanners called barcode readers. In the supermarket, there are many hand-held scanners that automatically scan the barcodes. In this paper work was done on 1D and 2D barcodes to detect them from multiple products with better efficiency and accuracy [2]. From [2], it is noted that they worked on mathematical morphology. In this proposed method doesn't have this type of morphological issue. Now a days, mobile phones/digital cameras are available everywhere and it is quite cheap according to the barcode scanners. So, this kind of method in such device like mobile phone will much more cost efficient.

The examples of 1-D barcode are Code 128, Code 39, EAN-8, EAN-13, UPC-A, UPC-E, I-25 etc. And Data Matrix, QR
Code, PDF417, MaxiCode are some examples of 2-D barcodes. But among these, EAN-13 [3], Code 128 and QR codes [4] are mostly used. Some of 1D and 2D barcodes are shown in Fig.1.

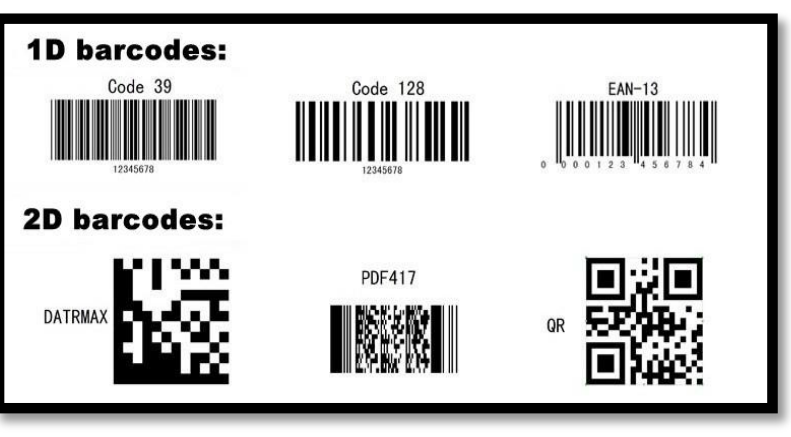

Fig.1: Some 1D \& 2D barcodes

There are mainly two tasks for barcode readers: barcode locating and barcode decoding [5]. This processes were implemented by using Python. Firstly an algorithm to detect the barcode from the snap were processed, then it is needed to decode it. And the main benefit in this process is, it is possible to detect multiple barcodes at a time and can detect the barcode of cylindrical objects too. Zamberletti algorithm was applied using opencv and pyzbar library to detect and decode the barcodes from the snap.

Now in this living era where everything, every process in each sector are being automated. Advantages commonly attributed to automation include higher production rates and increased productivity, more efficient use of materials, better product quality, improved safety, shorter workweeks for labor and reduced factory lead times. So, to make the maximum profit from a business it must be follow the trend which means it must be adopt automation and makes the highest and proper use of it. Automation requires much less or not at all human interaction. But traditionally in super shops used to see a lot of human interactions are made. People buy products. Then make one or more queue for scanning those products where some employees are appointed for scanning barcodes with a barcode scanner. It's a traditional view of a super shop. Here, the authors of this paper focused on two things. Firstly, a lot of time is wasted of both consumers and sellers for the scanning method. Moreover, the barcode scanner which is normally used is very much. If any people start an ordinary super shop but wants to put in barcode system in his shop the costing will be inappropriate for him. So, these factors can be overcome if there is something else instead of that barcode scanners and be able to scan multiple products simultaneously. In this way it is easily plausible to minimize the cost and can get rid of from long time spending at queue. 
That's how this idea can play a great role to maximize the profit in a business. In this paper, section 2 describes the related study on this topic. Section 3 and 4 describe about our methodology and results. Challenges and Conclusion are described in section 5 and 6 .

\section{RELATED STUDY}

In this section, we will discuss about our background study and works.

\subsection{Overview of Barcode}

A barcode is "A machine-readable code in the form of numbers and a pattern of parallel lines of varying widths, printed on and identifying a product." A bar code can best be described as an "optical Morse code." Series of black bars and white spaces of varying widths are printed on labels to uniquely identify items. The bar code labels are read with a scanner, which measures reflected light and interprets the code into numbers and letters that are passed on to a computer. Because there are many ways to arrange these bars and spaces, numerous symbolisms are possible. But in truth a barcode is so much more. Barcode systems help businesses and organizations track products, prices, and stock levels for centralized management in a computer software system allowing for incredible increases in productivity and efficiency. There are two types of barcodes: linear - or 1D, and 2D. Mostly recognizable barcode visually, the UPC (Universal Product Code) is a linear 1D barcode which consists of two parts: the black and white pattern recognized as barcode and the 12-digit UPC number. The first six digits of the barcode represents the pattern as well as the manufacturer's identification number. The next five digits represent the item's number. The last digit is termed as a check digit which helps the scanner to determine if the barcode being scanned is free of error.

The 1D barcode has contains many things as it has a unique structure. The structure contains:

- Margin: Spaces, normally white, where nothing printed are required at each end of the code and they should be 10 times that of a narrow bar.

- Barcode symbol: The area composed of bars and spaces is known as the "bar code symbol".

- Barcode: The symbol together with the left and right margins make up what is called bar code.

- Start character: Indicates the start of the data. Depending on code system, start character varies.

- Data(message): Area indicating the actual data.

- Check digit: Confirms that there is no error in reading by means of one digit of compiled data.

- Stop character: Indicates the end of the data.

A barcode's density is determined by the " $\mathrm{X}$ " dimension. Density refers to the amount of information that can be captured in the bar code in a particular space, usually a linear inch. While not intuitively obvious, high density bar codes have low numbers and low density bar codes have high numbers. This is because individual characters consist of some combination of bars and spaces that are each multiple of " $X$ ". When " $X$ " is small, the area required for each character is less than when " $\mathrm{X}$ " is large; thus the bar code can hold more per linear inch and is said to be of higher density. Similarly, increasing the width of the narrowest element ("X") increases the space required for each character and reduces the number of characters per inch. Because the resulting code is often quite large, very low density codes are often associated with applications such as warehousing that require reading bar codes from a significant distance ( 3 to 30 feet).

\subsection{Detection Policy from Previous Works:}

Day by day we are going towards an era in which every process is being automated. This means much less or not at all human interaction. But traditionally super shop billing system requires a lot of human interaction. Again, the barcodes scanner's costing is much higher according to their longevity. And most importantly, the work of multiple barcode detection from an image was done on 1D barcodes and they are not angle invariant. There was a method of extracting information from the barcode at a lesser cost compared to typical electronic barcode scanners [1]. They had analyzed their diagram by following three levels, they are: level 0 , level 1 , level 2 . They saved the product information in database. Then the barcode was scanned and decoded and then, after matching the product information, the product bill had been updated. EAN-13 barcode was used for this experiment. After that, if we take a look, then we see that there was another experiment on barcodes, especially single and plural barcodes which were detected at a time and the accuracy was 0.7 seconds to detect the barcodes [5]. The method was to extract candidate barcode skeletons to locate barcodes in ana image. The method was capable of searching rotated barcodes in a high-resolution image. Another work was to detect QR codes from cylindrical object like bottles, cans [6]. These works influences us to work on 1D and 2D barcodes at a time that means multiple barcode detection. The papers also represented the angle invariant barcode detections.

If we give a look, then we can see that there was a paper on detecting barcodes through cross identification using mobile platform [7]. The paper included the X-shaped and T-shaped crosses, filtered false crosses and then detected the barcodes. Experimental results show the efficiency and robustness of the proposed method in the presence of blurry and complex background of scene images. So, the processes that are implemented before were not about the combination of single and multiple 1D, 2D barcodes and angle invariant barcodes. That's why we are focusing on this so that we can implement all the tasks in one process. Another paper presents a method for making the barcode identification system more efficient [8]. They worked on identifying QR and AZTEC code. Firstly, captured color image is converted into a gray scale. Then they binarized it by Ostu's Algorithm. The CCL (Connected Component Labeling) Algorithm was carried out to obtain the tag image. A paper proposed a method of identifying the QR and Aztec barcodes by using connected component labeling algorithm to compute the tag for each connected component, then search for the innermost connected component of the finder patterns in the tag image [8]. The method can identify rotated or defocused barcode images. Another work has been done on making this system angle invariant and less or not at all user interactive [9].

Nowadays mobile applications have been using to identify products from pictures and also that can make online comparisons with the existing similar products. This approaches mainly focus on decoding degraded barcodes and treat the underlying barcode detection task as a side problem that can be addressed using appropriate object detection methods. However, the majority of modern mobile devices do not meet the minimum working requirements of complex 
general purpose object detection algorithms and most of the efficient specifically designed barcode detection algorithms require user interaction to work properly. But in this work, they presented a novel method for barcode detection in camera captured images based on a supervised machine learning algorithm that identifies one dimensional barcodes in the two-dimensional Hough Transform space and remarkably it is angle invariant, requires no user interaction and can be executed on a modern mobile device. Firstly, in this process, the rotation angle of the barcode is determined with Hough space using Multi-Layer Perceptron (MLP). Then, determine the set of all the segments of the image by applying the same technique of Matasyz [10]. After that, two histograms are defined that describe the intensity profile of the rows and the columns. Finally, a smoothing filter is applied to each histogram to remove low value bins corresponding to isolated non-barcode segments and the bounding box of the barcode is determined at last.

\subsection{Barcode Recognition Policy:}

A linear barcode typically holds any type of text information. In contrast, a 2D barcode is more complex and can include more information in the code: price, quantity, web address or image. A linear barcode scanner cannot read a 2D barcode, requiring the use of an image scanner for reading the information embedded in a 2D barcode. Mobile phones with cameras, like iPhones and Android phones, and many other devices can read 2D barcodes through their integrated cameras. There are mainly two tasks for barcode readers: barcode locating and barcode decoding. Barcode localization methods have two objectives, speed and accuracy. For industrial environment, accuracy is crucial since undetected (missed) codes may lead to loss of profit. Processing speed is a secondary desired property of the detectors. On smartphones, the accuracy is not so critical, since the device interacts with the user and re-shooting is easily possible, but a fast (and reasonably accurate) barcode detection is desirable. Various techniques are used to locate and decode barcodes from photographs, from the classical line scanning technique [11], through the widely studied morphological approaches [12]. Once an image is obtained, the first step is to localize the barcode, or to find its location within the image. Many methods to do this have been developed. Again, after the barcode is localized using one of the methods, it must be decoded in order to obtain the product's information.

\section{PROPOSED METHODOLOGY}

Barcode detection system is very important for all kinds of shop, so that the products of the shop can be arranged in a better way. After reviewing all of these papers, it has come to mind that there were many scopes to improve the method for barcode detection in many ways. Firstly, multiple 1D barcode detection was initial target. Besides fulfilling the target search was on for better filtering option without degrading the accuracy. Barcode detection on complex background was part of work.

In shopping system, it is observed that there is a laser scanner for detecting barcodes from a product. But if there are multiple products, then the system need more time to detect many products. And it is tough to detect multiple product barcodes one by one at a time. So, our method is helpful for solving this issue so that it takes less time in shopping system to detect all the barcodes. This proposed method is to detect multiple 1D and 2D barcodes from a snap. EAN-13, Code 128 as 1D barcode and QR Code as 2D barcode were used. For the detection of the barcodes, two steps were followed. They are:
- To find the position of a barcode

- To decode the barcode.

Once an image is obtained, the first step is to localize the barcode, or to find its location within the image. Many methods to do this have been developed. Again, after the barcode is localized using one of the methods in the previous section, it must be decoded in order to obtain the product's information. These processes were achieved by the usage of Python language. Firstly, an algorithm was processed to detect the barcode from the snap, then the job of decoding comes. And the main benefit in this process is, detecting multiple barcodes at a time and detection of the barcode of cylindrical objects too attainable. We have used Zamberletti algorithm using OpenCV and ZBar library to detect and decode the barcodes from the snap. There are three main methods of this proposed barcode detection and decoding process. They are: Preprocessing or Localizing, Barcode decoding and Barcode displaying.

\subsection{Preprocessing}

After taking an image as input, preprocessing is required this is just like resize the image, filtering the image. Initially image had the resolution of $3146 \times 3146$ pixels which was reduced to $528 \times 704$ pixels. The image is converted to grey scale image. Then converted to ZBar compatible format to find image symbols.

\subsection{Barcode decoding}

In this stage, the pyzbar library decodes the barcode. In the preprocessing stage image is already in ZBar compatible format. Then scanlines are used to iterate over the symbols to extract type, data and location information.

\subsection{Barcode displaying}

After decoding next step is displaying the symbols. A quad is formed outside the barcode while displaying 2D barcode image. If location is not a quad an outer boundary box is drawn. 

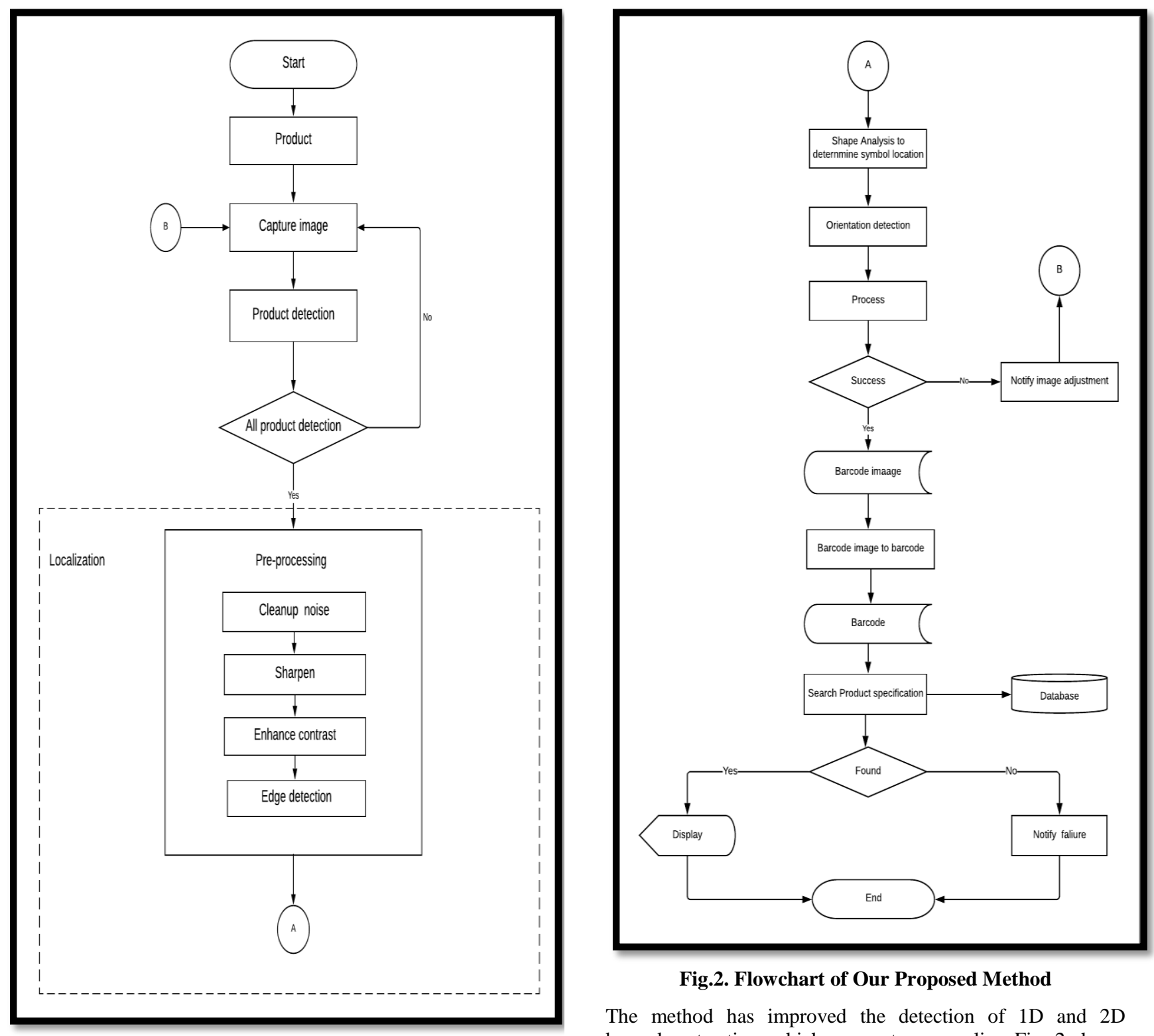

Fig.2. Flowchart of Our Proposed Method

The method has improved the detection of $1 \mathrm{D}$ and 2D barcodes at a time which was not easy earlier. Fig. 2 shows the flow diagram of our working process. There are many processes in this experiment such as: cleanup noise, sharpen the image, enhance the contrast, edge detection which are belonged to preprocessing system. After the shape analysis of the product, then the barcode detection process will work. This algorithm works fluently with the help of some python library.

\section{EXPERIMENTAL ANALYSIS}

\subsection{Implementation Process:}

For the detection and decoding of the barcodes, firstly the barcode(s) from a snap was detected and start the preprocessing. As ZBar Barcode Reader library was used, so this library enabled to improve the detection process of the barcodes. ZBar Barcode Reader library is an open source software suite for reading bar codes from various sources, such as video streams, image files and raw intensity sensors. It supports EAN-13/UPC-A, UPC-E, EAN-8, Code 128, Code 39, Codabar, Interleaved 2 of 5 and QR Code. Included with the library are basic applications for decoding captured bar code images and using a video device (eg, webcam) as a bar code scanner. Here the resolution plays an important role for 
the detection of the barcodes. For testing purpose, the dataset of ArTe Lab was accounted for in which the used the resolution of $1600 \mathrm{X} 1200$. Success rate decreased by compressing the resolution. For our prepared dataset resolution was fixed which was about $800 \times 600$. By fixing the resolution success rate was better. The decoding process is implemented by calling decode function that is a built-in function in python. "Type" and "Value" of the barcode(s) in decoding process is shown in some figures. The decoding process is important because in a shop, while buying the products, the product type and product value is important to match with the database for billing perfectly.

\subsection{Dataset:}

For the detection and decoding process of the barcodes, firstly analysis was done on some products which have 1D and 2D barcodes. There are various types of shapes and sizes of various types of products and various 1D barcode types which are shown in Table 1.1.

Table.1: Dataset for detecting and decoding barcodes

\begin{tabular}{|c|c|c|c|}
\hline Product & Product Shape & Product Type \& Size & Barcode Type \\
\hline Cosmetic & $\begin{array}{l}\text { Quadruple } \\
\text { Cylindrical } \\
\text { Cylindrical } \\
\text { Rectangular } \\
\text { Curved Rectangular } \\
\text { Round } \\
\text { Curved Quadruple } \\
\text { Cylindrical }\end{array}$ & $\begin{array}{l}\text { Facewash (Small) } \\
\text { Facewash (Big) } \\
\text { Lipstick (Small) } \\
\text { Lipstick (Small) } \\
\text { Lotion (Big) } \\
\text { Cream (Big) } \\
\text { Cream (Big) } \\
\text { Oil (Small) }\end{array}$ & $\begin{array}{l}\text { EAN-13(1D) } \\
\text { EAN-13(1D) } \\
\text { EAN-13(1D) } \\
\text { EAN-13(1D) } \\
\text { EAN-13(1D) } \\
\text { EAN-13(1D) } \\
\text { EAN-13(1D) } \\
\text { UPC-A(1D) }\end{array}$ \\
\hline Food & $\begin{array}{l}\text { Curved Rectangular } \\
\text { Cylindrical } \\
\text { Square } \\
\text { Rectangular }\end{array}$ & $\begin{array}{l}\text { Spices (Big) } \\
\text { Can (Big) } \\
\text { Chips (Big) } \\
\text { Chocolate (Big) }\end{array}$ & $\begin{array}{l}\text { EAN-13(1D) } \\
\text { EAN-13(1D) } \\
\text { EAN-13(1D) } \\
\text { EAN-8(1D) }\end{array}$ \\
\hline Stationary & $\begin{array}{l}\text { Square } \\
\text { Rectangular } \\
\text { Cylindrical } \\
\text { Rectangular }\end{array}$ & $\begin{array}{l}\text { Book (Big) } \\
\text { Notebook (Big) } \\
\text { Marker (Small) } \\
\text { Stapler (Small) }\end{array}$ & $\begin{array}{l}\text { Code-39(1D) } \\
\text { Code-39(1D) } \\
\text { UPC-A(1D) } \\
\text { EAN-13(1D) }\end{array}$ \\
\hline Beverage & $\begin{array}{l}\text { Cylindrical } \\
\text { Curved Rectangular } \\
\text { Cubic }\end{array}$ & $\begin{array}{l}\text { Can (Big) } \\
\text { Bottle (Small) } \\
\text { Packet (Small) }\end{array}$ & $\begin{array}{l}\text { EAN-13(1D) } \\
\text { EAN-13(1D) } \\
\text { EAN-13(1D) }\end{array}$ \\
\hline Electronic Accessories & $\begin{array}{l}\text { Rectangle } \\
\text { Square }\end{array}$ & $\begin{array}{l}\text { Mobile Packaging (Big) } \\
\text { Speaker Packaging (Big) }\end{array}$ & $\begin{array}{l}\text { EAN-13(1D) } \\
\text { EAN-13(1D) }\end{array}$ \\
\hline
\end{tabular}

Next, the dataset of ArTe Lab [13] was part of experiment. Here the resolution is one of the important facts because if the resolution is high, then the detection accuracy might be high. Sometimes it varies. So, the standard resolution for us is 800 X 600. Table.2 demonstrates the detection accuracy of ArTe Lab.

Table.2: Arte database: Resolution vs Accuracy

\begin{tabular}{|l|l|}
\hline Resolution & Accuracy \\
\hline $1600 X 1200$ & $89.90 \%$ \\
\hline $784 X 588$ & $71.60 \%$ \\
\hline
\end{tabular}

Then timing needed for detecting the barcodes was checked that is shown in Table.3.

Table.3: Arte database: Resolution vs Time

\begin{tabular}{|l|l|}
\hline Resolution & Time \\
\hline $1600 X 1200$ & 4 minutes 48 seconds \\
\hline 784 X588 & 2 minutes 10 seconds \\
\hline
\end{tabular}




\subsection{Detection Results:}

The experiment has shown the result of single and multiple barcodes. The barcodes are detected and decoded taking a less time. The analysis of barcode is done by ZBar library of python. First of all, the algorithm was applied with single computer generated 1D barcode and got the positive result of detection shown in Fig.3.
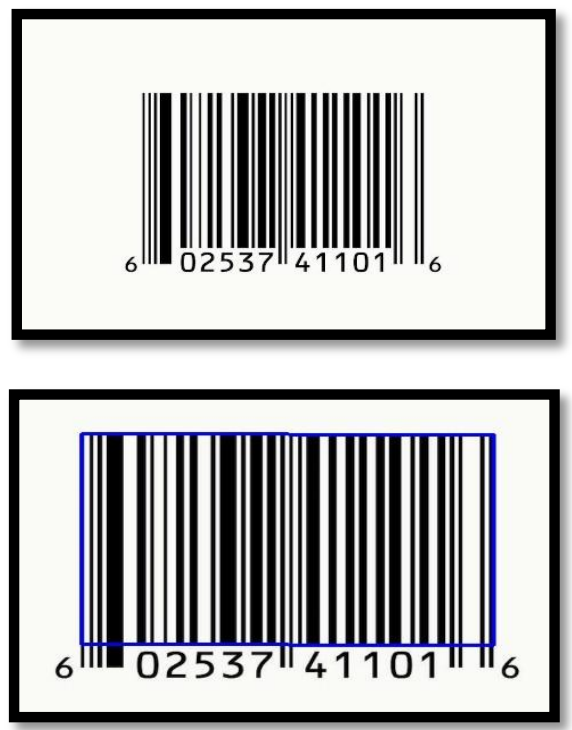

Fig.3: Single barcode detection

Then detection of single barcode from an image was tried. And the barcode was detected successfully. Fig.4 illustrates the result.

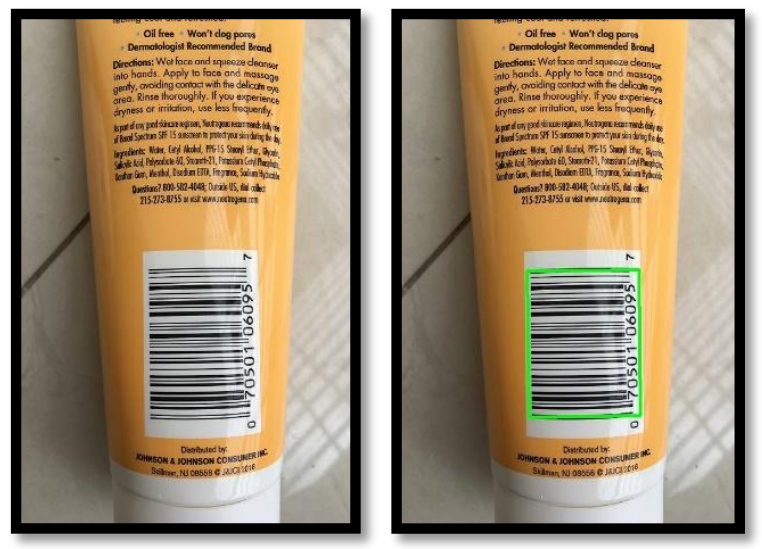

Fig.4: Single barcode detection from an image

Next target was to detect multiple barcodes at a time. Again, computer generated multiple barcodes for the experiment was used. From Fig.5, we see that there are multiple barcodes in one frame. Here, the data matrix is not barcode, so it can't be detected by ZBar library.
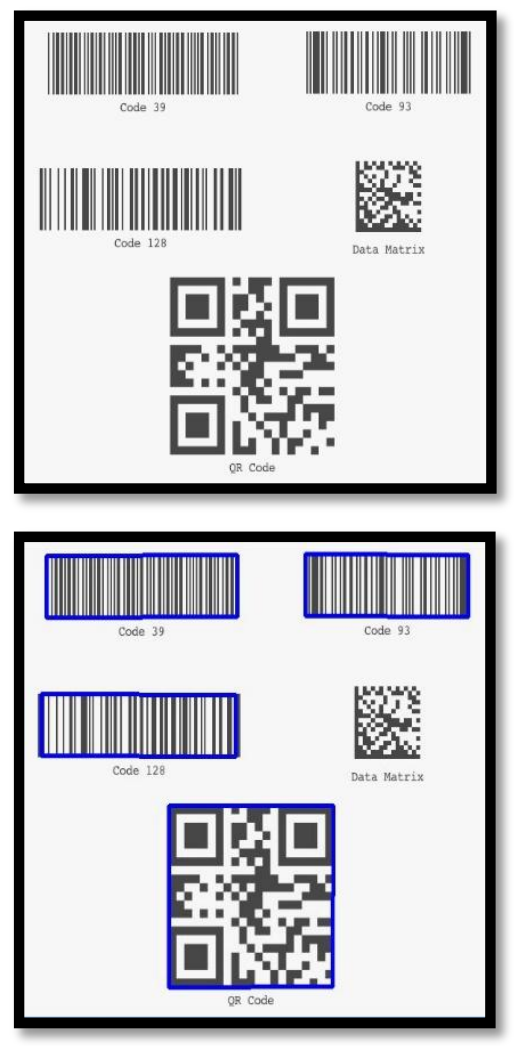

Fig.5: Multiple barcode detection

Barcode detection part worked successfully with single, multiple $1 \mathrm{D}$ and $2 \mathrm{D}$ barcodes. It took less time to detect all the barcodes in an image. After doing the process, our challenge was to decode them from an image. The proposal was to detect and decodes the barcode from real time image therefor a snap captured from mobile. So then detection process from real time image was tried hence a mobile image. There were multiple barcodes in a complex background that works easily for detection. Fig.6 shows the white background and some products to detect barcodes from them.

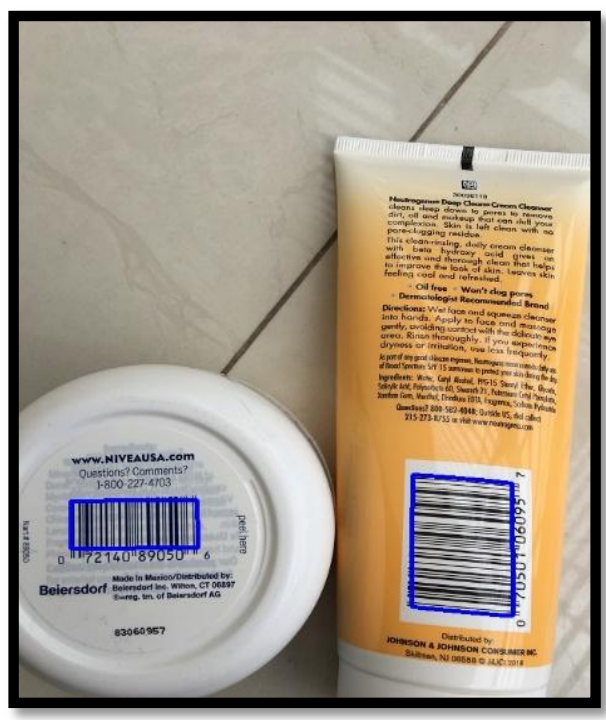

Fig.6: Multiple barcode detection from mobile snap

Testing the barcodes in white background may seem like an easy step. That's why we targetted to detect the barcode in 
complex background, and detected that barcode easily. Fig.7 illustrates this result.

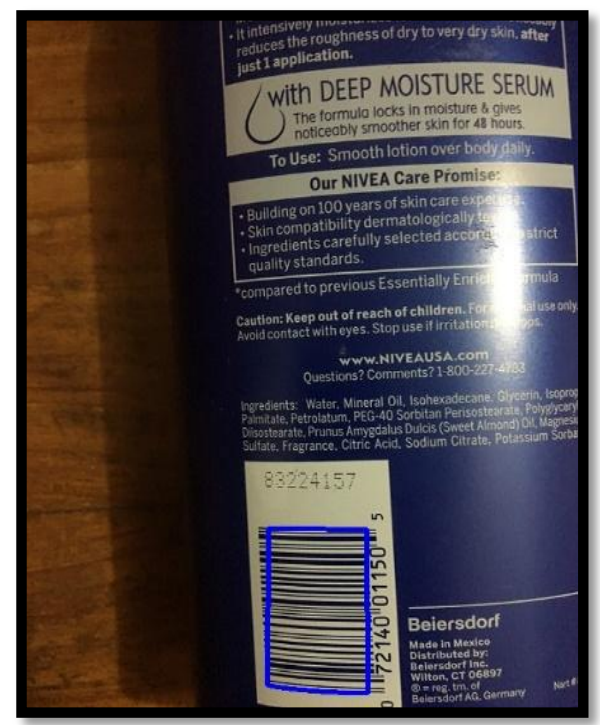

Fig.7: Single barcode detection from mobile snap

The barcode detection process from a normal snap was done successfully. So it was tough for detectiong the barcodes which are angle invariant. The next step was detecting the barcodes which are angle invariant and we got success at this stage too (Fig.8). The degree is about " +-5 " degree to detect angle invariant barcodes.

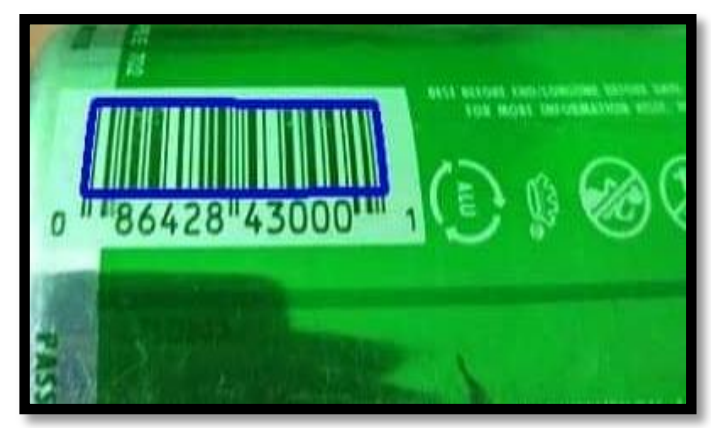

Fig.8: Angle invariant barcode detection

After completing the part of 1D barcode detection, Then to detect the 2D barcodes like QR code was attempted. The QR code was also detected which is shown in Fig.9.

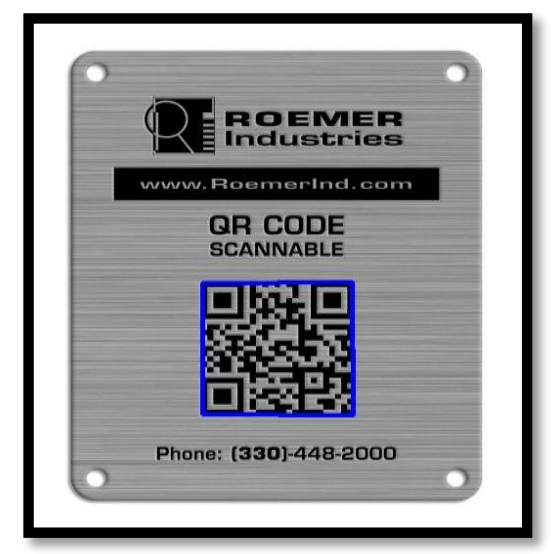

Fig.9: Single 2D barcode detection from mobile image
Barcode detection process was done by zbar library. But this library has another feature to decode the barcode at the same time. For decoding the 1D barcodes, The type and data of the barcodes that are shown in Fig. 10.

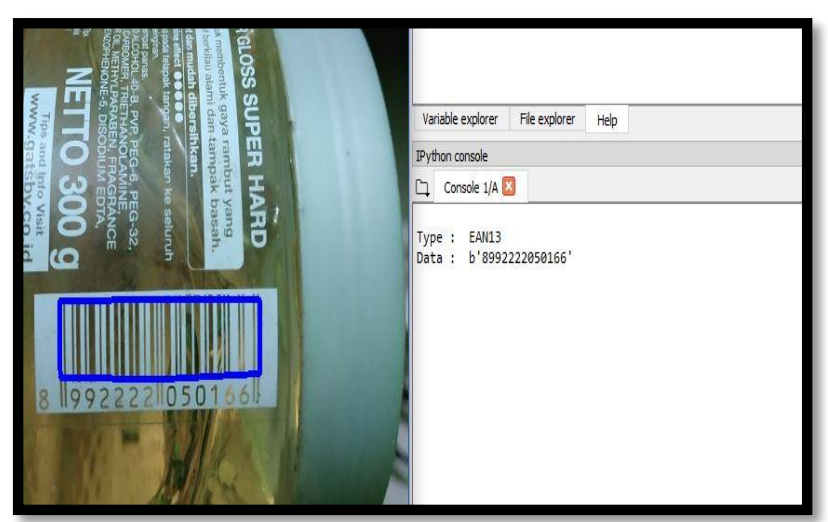

Fig.10: Detecting and decoding single 1D barcode from a snap

The decoding of 2D barcodes as QR code is shown in Fig.11.

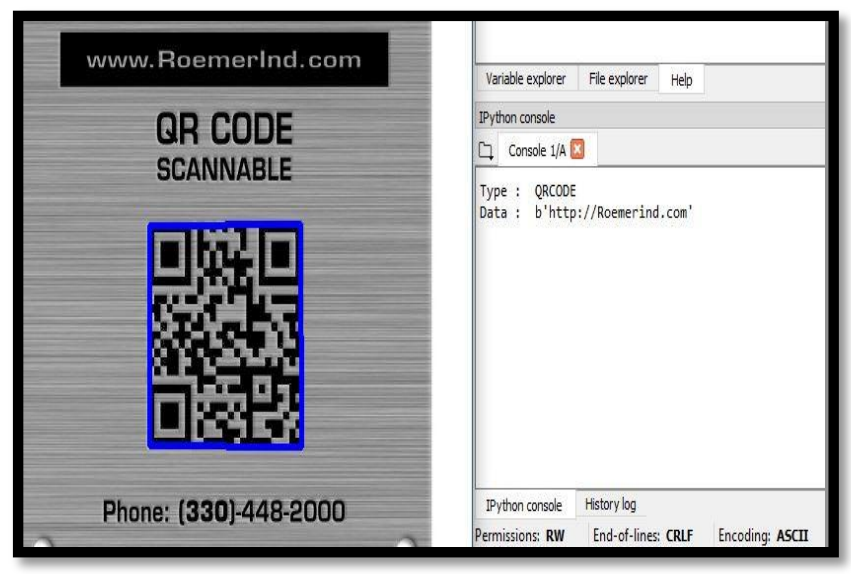

Fig.11: Detecting and decoding single 2D barcode from an image

Multiple barcode decoding was not easy as single barcode decoding. But we got succes too at this stage. Fig.12 shows this part.

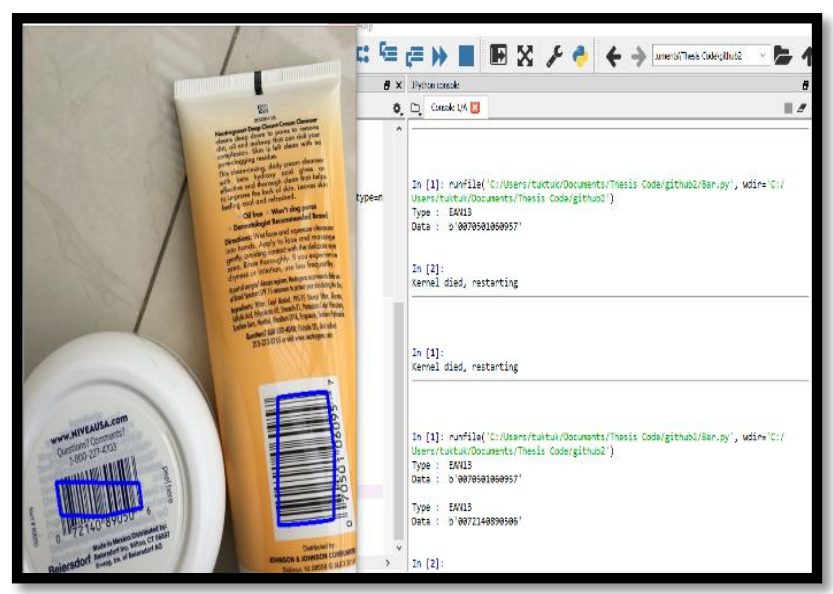

Fig.12: Detecting and decoding multiple barcodes from a snap 
Last of all, we can ensure that our method will work for detecting and decoding any kind of barcodes from a snap with proper resolution. The simple and complex background doesn't matter to detect and decode the barcodes.

\section{CHALLENGES}

Though detection of single $1 \mathrm{D}$, single $2 \mathrm{D}$ and multiple barcodes was accomplished, but this method always didn't provide correct result. Some factors such as noise, reflection, contrast, unfocused image etc. cause some problems while detecting the barcode. For single 1D and single 2D barcode most of the time accurate result was delivered but sometimes because of lack of lighting, reflection, blur image was causing failure in detection. While detecting single 1D and 2D, it was possible to detect the barcode from low resolution images and that was helpful for shorten the time of detection. But for the detection of multiple barcodes, it always requires high resolution images and sometimes in spite of having high resolution, some barcodes remain undetected because of having tiny or uncommon shapes. It happens in both our dataset and Arte-lab-dataset.

\section{CONCLUSION}

Barcode is now available for every product. To detect barcodes, if one totally sums up, he will see a huge amount of working hours is used. If in barcode detection process of a superstore, multiple products can be included at a time and with great efficiency and higher accuracy, then it will save huge time. It will be beneficial for a shop if they take less time for their product management and billing system. Thus the productivity will increase. Again, making a system less user interactive or no user interactive at all makes the whole process more functional. Some of the people worked on single 2D barcode, some on multiple 2D barcode. Further, some people also worked on detecting angle invariant multiple 1D barcode. But, we have tried to implement all of those issues in one platform and obviously through mobile camera sensor. Hope, it will be much lucrative in business sectors.

\section{REFERENCES}

[1] [A. N. K. A. U. N. R. A. M. R. Sampada S. Upasani, "Robust Algorithm for Developing Barcode Recognition System using Web-cam," International Journal of Scientific \& Engineering Research, vol. 7, no. 4, pp. 8286, April 2016.

[2] K. V. Gowtham M N, "Generating EAN-13 Standard Barcodes," International Journal of Science and Research (IJSR), vol. 3, no. 6, pp. 1307-1309, 2014.
[3] M. K. a. L.'.' G. N.'ul, "Efficient 1D and 2D barcode detection using mathematical morphology".

[4] H. J. G. Kinjal H. Pandya, "A Survey on QR Codes: in context of Research and Application," International Journal of Emerging Technology and Advanced Engineering, vol. 4, no. 3, pp. 258-262, 2014.

[5] K.-T. W. M.-C. W. N.-Y. C. J.-H. W. Chun-Shun Tseng, "Retrospective Tracking for Barcode Reading," IEEE, pp. 114-119, 2010.

[6] Z. S. D. S. H. Xiaochao Li, "Reconstruct Algorithm of 2D barcode for Reading the QR Code on Cylindrical Surface," The Natural Science Foundation of China.

[7] Y. Z. a. T. Lu, "A Fast Color Barcode Detection Method through Cross," 13th International Conference on Document Analysis and Recognition (ICDAR), pp. 416420, 2015.

[8] S.-C. L. a. P.-H. Wang, "Design of a barcode identification system," Consumer Electronics-Taiwan (ICCE-TW), IEEE International Conference, pp. 237238, 2014.

[9] I. G. a. S. A. Alessandro Zamberletti, "Robust angle invariant 1d barcode detection," 2nd IAPR Asian Conference, pp. 160-164, 2013.

[10] C Galamhos, Jose Matas, and Josef Kittler. "Progressive probabilistic hough transform for line detection," Computer Vision and Pattern Recognition, 1999. IEEE Computer Society Conference on., volume 1, pages 554560. IEEE, 1999.

[11] Robert Adelmann, Marc Langheinrich, and Christian Floerkemeier. "Toolkit for bar code recognition and resolving on camera phones-jump starting the internet of things," GI Jahrestagung (2), 94:366-373, 2006.

[12] Timothy R Tuinstra. "Reading barcodes from digital imagery," $\mathrm{PhD}$ thesis, $\mathrm{PhD}$ thesis, Cedarville University, 2006.

[13] Alessandro Zamberletti, Ignazio Gallo, Moreno Carullo and Elisabetta Binaghi. "Neural Image Restoration For Decoding 1-D Barcodes Using Common Camera Phones," Computer Vision, Imaging and Computer Graphics. Theory and Applications, Springer Berlin Heidelberg, 